\title{
RENTABLES, UMWELTFREUNDLICHES UND GESELLSCHAFTSDIENLICHES WIRTSCHAFTEN IST MÖGLICH. MEHR NACHHALTIGKEIT DANK EINER BESSEREN OPERATIONALISIERUNG VON CORPORATE SOCIAL RESPONSIBILITY
}

\begin{abstract}
Zwar macht der Begriff „soziale Verantwortung von Unternehmen“ in der Öffentlichkeit heute Furore, bedauerlicherweise wird er jedoch sehr inflationär verwendet, was dem eigentlichen CSR-Anliegen auf Dauer wenig nützt. Gleichzeitig wird im Zusammenhang mit den allgegenwärtigen Nachhaltigkeitsslogans eine tiefgreifende Werteumorientierung Re-Moralisierung und Re-Sozialisierung der Wirtschaft - postuliert. Worin sozial verantwortliches Unternehmenshandeln genau besteht, ist jedoch heftig umstritten. In der Folge dieser semantischen Verwirrung wird in der Diskussion immer lauter gefordert, auf den CSR-Begriff als unbrauchbar oder irreführend ganz zu verzichten und ihn durch differenziertere Bezeichnungen zu ersetzen. ${ }^{2}$ Im vorliegenden Beitrag wird versucht, den CSR-Begriff zu verteidigen und zu zeigen, dass es nicht nur sozialethisch, sondern auch wirtschaftswissenschaftlich gute Gründe gibt, ihn aufrecht zu erhalten. Die zentralen Thesen der Ausführungen lauten: Ein nachhaltiges, umweltfreundliches und gesellschaftsdienliches Wirtschaften ist möglich. Auf der Ebene des Unternehmens führt der Weg dahin über eine ganze Fülle von möglichen CSR-Aktivitäten, die von den Unternehmen allerdings noch nicht vollständig erkannt werden. Anhand der in der Richtlinie ISO 26000 gegebenen Orientierung wird das Leitbild der Corporate Social Responsibility auf sechs Ebenen operationalisiert: (1) der Unternehmensführung, Organisation und Managementsysteme, (2) der Beschäftigungspolitik, (3) des Umwelt- und Ressourcenmanagements, (4) der Interaktionen mit dem wirtschaftlichen Umfeld, (5) der Relationen mit Kunden und Verbrauchern sowie (6) der Interaktionen mit der Öffentlichkeit und lokalen Gemeinschaften. Dank der CSR-Operationalisierung nimmt die Vision eines nachhaltigen Wirtschaftshandelns eine etwas konkretere Gestalt an.

Schlüsselbegriffe: Nachhaltigkeit, soziale Verantwortung von Unternehmen, Operationalisierung, Unternehmensethik.
\end{abstract}

\section{EINLEITUNG}

Die Entwicklung der neuen Verantwortungskonzepte in der Wissenschaft, die eine Herausbildung neuer Typen zwischenmenschlicher Solidarität und moralischer Sensibilität in der Gesellschaft begleiten, hängt mit den aktuellen politischen,

\footnotetext{
${ }^{1}$ Dr Krzysztof Michalski, Zakład Nauk Humanistycznych, Wydział Zarządzania, Politechnika Rzeszowska, ul. Poznańska 1, 35-084 Rzeszów, tel. 1786512 04, e-mail: michals@prz.edu.pl ${ }^{2}$ Vgl. I. Ślęzak-Gładzik, Corporate Social Responsibility (CSR) jako koncepcja porządkująca relacje między biznesem a społeczeństwem [CSR als ein die Beziehungen zwischen Wirtschaft und Gesellschaft ordnendes Konzept]. In: Modern Management Review, Vol. XVIII, No. 2(2013), S.115 (poln.).
} 
wirtschaftlichen und soziokulturellen Globalisierungsprozessen eng zusammen. Angesichts der Fortschritte in Wissenschaft und Technik und der Hegemonie der ökonomischen Prozesse, die für eine massive weltweite Diffusion der „Früchte“ des Fortschrittssorgen, wächst das individuelle und allgemeingesellschaftliche Bewusstsein über die mit dem Fortschritt einhergehenden Gefahren für den Menschen, die Gemeinschaft und die gesamte Menschheit. Einerseits gibt eine fortschreitende Umweltzerstörung, tiefgreifende soziale Umstrukturierungen und die Herausbildung neuer Machtverhältnisse sowie zunehmenden Verlust gesellschaftlicher Kontrolle über die zivilisatorischen Entwicklungsprozesse durch eine enorme Steigerung ihrer Komplexität, andererseits ermöglichen Entwicklungen in der Wissenschaft, die u.a. dank der Erarbeitung von Methoden und Verfahren einer umfassenden Früherkennung der Zukunft und Folgenabschätzung dieser zivilisatorischen Prozesse, einen verantwortungsvollen gesellschaftlichen Umgang mit eben dieser Komplexität. Neben den kognitiven Kompetenzen, um die wichtigsten Folgen und Auswirkungen unseres Handelns frühzeitig und räumlich wie zeitlich weitreichend erkennen $\mathrm{zu}$ können, rücken auch gewisse normative und operationale Kompetenzen, um diese Folgen auf Human-, Sozial- und Umweltverträglichkeit hin beurteilen und durch politisch-organisationale Maßnahmen auf allen relevanten Ebenen des gesellschaftlichen Lebens den gesellschaftlich anerkannten Gerechtigkeitsvorstellungen entsprechend gestalten zu können, in den Vordergrund. Eine wichtige Rolle spielen dabei die veränderten Vorstellungen von Bezügen, Inhalten und Reichweiten moralischer und sozialer Verantwortung. ${ }^{3}$ So betrachten wir beispielsweise dank der verbreiteten Nutzung von Internet und Satellitenfernsehen ferne Probleme fremder Menschen zunehmend als unsere eigenen Anliegen und identifizieren uns immer häufiger mit Menschen aus fernen Ländern, die unter Hunger, Verfolgung oder Ungerechtigkeit leiden. ${ }^{4}$ Dies führt zu einer allmählichen, aber äußerst tiefgreifenden Umorientierung sowohl in der individuellen als auch in der gesellschaftlichen Wahrnehmung von moralischen Pflichten und aktuellen Erfordernissen moralisch, ökologisch und sozial verantwortlichen Handelns. Unter diesen Umständen stehen die Chancen der im vorliegenden Beitrag postulierten Ethik einer Langzeit- und Langstreckenverantwortung ${ }^{5}$, die sich auf der Ebene der Unternehmen vor allem in CSR-

\footnotetext{
${ }^{3}$ Siehe hierzu H. Jonas, Prinzip Verantwortung. Versuch einer Ethik für die technologische Zivilisation. Frankfurt am Main 1979; D. Birnbacher, Verantwortung für zukünftige Generationen. Stuttgart 1988; K. Bayertz (Hrsg.), Verantwortung: Prinzip oder Problem. Darmstadt 1995, M. Maring, Kollektive und korporative Verantwortung: Begriffs- und Fallstudien aus Wirtschaft, Technik und Alltag. Münster 2001.

${ }^{4}$ Vgl. J. Bhagwati, In Defense of Globalization. New York 2004, S. 19; E. Bińczyk, Technonauka w społeczeństwie ryzyka. Filozofia wobec niepożądanych następstw praktycznego sukcesu nauki [Technowissenschaft in der Risikogesellschaft. Die Philosophie angesichts unerwünschter Folgen des praktischen Erfolgs der Wissenschaft]. Torun 2013, S. 374 (poln.).

${ }^{5}$ Der Begriff der „Langstreckenverantwortung“ ist eine Ergänzung zum Begriff der Langzeitverantwortung und bezieht sich parallel zur Langzeitverantwortung sowohl auf die räumlich versetzten Handlungsfolgen, d.h. sekundäre und tertiäre Effekte unseres Handelns, die oft Menschen auf anderen Erdteilen betreffen, als auch auf räumlich erweiterte Wirkungspotentiale unseres Handelns in Zeiten der fortschreitenden Globalisierung im allgemeinen. Vgl. K. Abriszewski, Interpretacja i etyka dalekiego zasięgu [Interpretation und Ethik einer Langzeit- und Langstreckenverantwortung]. In: A. F. Kola, A. Szahaj (Hrsg.), Filozofia i etyka interpretacji [Philosophie und Ethik der Interpretation]. Kraków 2007, S. 277-288 (poln.).
} 
Initiativen und nachhaltiger Produktion niederschlägt und Individuen zum bewussten, verantwortungsvollen und nachhaltigen Konsumverhalten bewegt, gar nicht schlecht.

Vor diesem Hintergrund wird im Folgenden für eine Einbeziehung von Initiativen im Bereich sozialen Engagements und gesellschaftlicher Verantwortung von Unternehmen in die umfangreichen Bemühungen um die Verwirklichung Nachhaltiger Entwicklung plädiert. Die bisherigen Aktivitäten auf diesem Feld gelten als nur zum Teil erfolgreich. Es ist zwar gelungen, auf der internationalen politischen Ebene gewisse allgemeine Ziele $\mathrm{zu}$ definieren, diese durch bereichsspezifische Strategien zu operationalisieren und transnational, national wie regionalweitgehend umzusetzen, ${ }^{6}$ allerdings wurde im Rahmen der bisher entwickelten Strategien der Nachhaltigkeitsumsetzung auf den zentralen Gedanken aus dem Agenda 21 „Denke global, handle lokal“ zurückgegriffen und zur Grundorientierung für Privatpersonen, Gemeinschaften und Verwaltungseinheiten erklärt. Dabei wurden sehr wichtige Kapazitäten und Gestaltungspotenziale übersehen, die im Zuge steigender Dominanz der Wirtschaftsprozesse in der Handlungsbesonderheit und Wirkungsmacht moderner Unternehmen liegen. Angesichts zunehmender Reichweite, Kapitalkonzentration und Einflussnahme auf die Politik und das Leben einzelner Menschen sind hier die Möglichkeiten großer transnationaler Wirtschaftskorporationen und Konzerne besonders interessant. Laut Forschungsergebnissen der Weltbank sind unter den 100 weltgrößten und weltmächtigsten Wirtschaftsorganismen nur noch 48 Staaten, den bescheidenen „Rest“ von $52 \%$ machen globale Korporationen aus. ${ }^{7}$ Damit treten neben Institutionen der globalen und kontinentalen Politik wie die UNO mit allen ihren Agenturen, die Weltbank oder die OECD auch viele Wirtschaftsmächte auf die Weltbühne, die durch die Spezifik ihres Handelns die lokalen Problemlagen und Bedürfnisse besser erkennen und oft auch über bessere Möglichkeiten verfügen, diese Probleme auf eigene Faustdurch ehrlichere, gerechtere und gesellschaftlich verantwortlichere Investitions-, Produktions- und Handelspraxen und entsprechendes soziales wie ökologisches Engagement auf der globalen oder transnationalen Ebene zu lösen. Dieser Wandel der globalen Machtverhältnisse und die steigende Bedeutung des Unternehmenshandelns für das Alltagsleben des Einzelnen, der Gemeinschaft und der gesamten Gesellschaft muss bereits bei der Konkretisierung und Operationalisierung von Nachhaltigkeit wie auch der bei der strategischen Planung der Nachhaltigkeitsumsetzung angemessen berücksichtigt werden.

\section{CORPORATE SOCIAL RESPONSIBILITY UND NACHHALTIG} ENTWICKLUNG

Die Idee einer sozialen Verantwortung von Unternehmen und die Idee einer nachhaltigen Entwicklung sind Ausdruck und Erscheinung derselben tiefgreifenden soziokulturellen Wandlungsprozesse, die durch Globalisierung und Integration, Verwissenschaftlichung und Technisierung, Ökonomisierung und Ökologisierung sowie

\footnotetext{
${ }^{6}$ Eine aufschlussreiche Dokumentation dieser Entwicklungen in Mitteleuropa ist in der Nachhaltigkeits-Buchreihe des Verlags edition Sigma, Berlin, enthalten; siehe hierzu insbesondere G. Banse, A. Kiepas (Hrsg.), Nachhaltige Entwicklung: von der wissenschaftlichen Forschung zur politischen Umsetzung, Berlin 2005; G. Banse, R. Janikowski, A. Kiepas (Hrsg.), Nachhaltige Entwicklung - transnational. Sichten und Erfahrungen aus Mitteleuropa. Berlin 2011.

${ }^{7}$ Vgl. D. Barker, J. Mander, ABC globalizacji [ABC der Globalisierung]. Międzynarodowe Forum ds. Globalizacji [Internationales Forum für Globalisierung]. Łódź 2003, S. 23 (poln.).
} 
Demokratisierung des Lebens in den letzten 25 Jahren weltweit in Gang gesetzt wurden. Die Vision einer auf sozialer Gerechtigkeit in der intra- wie intergenerationellen Dimension fundierten nachhaltigen Entwicklung und einer neuen, auf zwischenmenschlicher Solidarität und Fürsorge basierenden prospektiven Verantwortung sind „moralisierende“ Antworten auf den weltweiten Orientierungsverlust angesichts der Krise, in die das Projekt Moderne geraten ist. Dabei stellen diese Antworten einen Versuch dar, dieses Projekt zu retten, ohne dessen Grundvoraussetzung hinsichtlich menschlicher Rationalität, Gutherzigkeit und Zurechnungsfähigkeit sowohl auf der Ebene des Individuums als auch auf der menschlicher Gemeinschaften bis hin zur Menschheit als Ganzes dabei aufgeben zu müssen. Hinter beiden Initiativen stehen im gleichen Maße Bemühungen, eine Makroethik globaler Gerechtigkeit, Solidarität und Verantwortung zu stiften. Auf die Genese, die Entwicklung, die Inhalte und zahlreiche Wechselwirkungen des Leitbildes nachhaltiger Entwicklung mit anderen Kulturträgern kann hier aus Platzmangel nicht näher eingegangen werden, es müssen Verweise auf die einschlägige Literatur ausreichend sein. ${ }^{8}$ Angesichts eines inflationären und polysemantischen Gebrauchs des Begriffes sozialer Verantwortung in der öffentlichen Debatte ist es dringlicher, hier das CSR-Konzept zu erörtern. Die Genese des Leitbildes einer sozialen Verantwortung von Unternehmen reicht bis in die frühen 1950er Jahre zurück und wird auf Arbeiten von Howard R. Bowen ${ }^{9}$ zurückgeführt. Angesichts der rapiden Konsumerismus-Entwicklung in den USA forderte Bowen eine umfangreiche Berücksichtigung des sozialen Umfelds und der Erwartungen der Gesellschaft beim Unternehmenshandeln. Die ursprüngliche Forderung, im Rahmen der sozialen Verantwortung die Interessen aller Betroffenen (Stakeholders)in die strategischen Entscheidungsprozesse im Unternehmen einzubeziehen, wurde später mit den Konzepten einer gesellschaftlichen Lizenz von Wirtschaftsorganisationen verknüpft und um andere Verantwortungsdimensionen erweitert. Daraus entstand das heutige, umfassende Verständnis von sozialer Verantwortung von Unternehmen, das viele unterschiedliche Facetten und gesellschaftliche Funktionen des Unternehmenshandelns ins Auge fasst. ${ }^{10} \mathrm{Im}$

\footnotetext{
${ }^{8}$ Ausführlich dazu O. Parodi, G. Banse, A. Schaffer (Hrsg.), Wechselspiele: Kultur und Nachhaltigkeit. Annäherungen an ein Spannungsfeld. Berlin 2010; G. Banse, G. L. Nelson, O. Parodi (eds.), Sustainable Development - the Cultural Perspective. Concepts - Aspects - Examples. Berlin 2011.

${ }^{9}$ Vgl. H. R. Bowen: Social responsibility of the businessman. New York 1953.

${ }^{10}$ In ihrer CSR-Richtlinie definiert die Europäische Kommission die soziale Verantwortung der Unternehmen ,als ein Konzept, das den Unternehmen als Grundlage dient, auf freiwilliger Basis soziale Belange und Umweltbelange in ihre Unternehmenstätigkeit und in die Wechselbeziehungen mit den Stakeholdern zu integrieren“. Stakeholder werden als „Anspruchsgruppen“ verstanden, also alle, die mit dem Unternehmen direkt oder indirekt in Beziehung stehen und aufgrund ihrer unterschiedlichen Interessen zu jeder Zeit Ansprüche an das Unternehmen erheben können: die Mitarbeiterinnen und Mitarbeiter, Kundinnen und Kunden, Zulieferbetriebe, Eigentümerinnen und Eigentümer usw., aber auch die Gesellschaft und der Staat. Die soziale Verantwortung der Unternehmen betrifft Maßnahmen, die die Unternehmen über ihre rechtlichen Verpflichtungen gegenüber Gesellschaft und Umwelt hinaus ergreifen; siehe hierzu Grünbuch Europäische Rahmenbedingungen für die soziale Verantwortung der Unternehmen. KOM(2001) 366 endgültig; Die EU-Richtlinie 2014/95/EU vom 22. Oktober 2014 zur CSR-Berichterstattung; ausführliche Darstellungen des CSR-Anliegens und der wichtigsten theoretischen Kontroversen finden sich in S. Hiß, Warum übernehmen Unternehmen gesellschaftliche Verantwortung? Ein soziologischer
} 
Zeitalter der nachhaltigen Entwicklung gewinnt das Leitbild sozialer Verantwortung und gesellschaftlichen Engagements von Unternehmen neue Bedeutungsdimensionen. Angesichts gemeinsamer Genese und verwandter Anliegen weisen die Leitbilder von Nachhaltigkeit und CSR weitgehende inhaltliche und strukturelle Ähnlichkeiten auf, die zu gleichen Problemen auf der Ebene der Rechtfertigung, Operationalisierung und Implementierung führen. Abgesehen von den Peripetien - d.h. jäh eintretenden entscheidenden Wendungen des bisherigen Handlungsverlaufs ins Gegenteil - bei der Implementierung beider kulturträchtigen Leitbilder unter konkreten gesellschaftlichen Rahmenbedingungen, auf die anderenorts eingegangen wird, ${ }^{11}$ können im Folgenden einige grundlegende Probleme im Hinblick auf die Operationalisierung des Leitbilds sozialer Verantwortung und gesellschaftlichen Engagements von Unternehmen thematisiert werden.

\section{OPERATIONALISIERUNG VON CSR}

Ähnlich wie die Idee einer nachhaltigen Entwicklung, die von einigen Kritikern für eine analytisch unvollendete und allzu abstrakte Sammlung von Slogans ${ }^{12}$ gehalten wird, liefert die Idee der sozialen Verantwortung und des gesellschaftlichen Engagements von Unternehmen lediglich eine unpräzise, vage Orientierung und Richtungsweisung, die notwendig mehrere Interpretationen und unterschiedliche, zum Teil gegensätzliche Visionen zulassen. Bei der Operationalisierung dieses Leitbildes kann man auf inhaltlichstrukturelle Vorgaben zurückgreifen, die in der Richtlinie ISO $26000^{13}$ enthalten sind. Als wichtige Referenzbereiche für die soziale Verantwortung von Unternehmen werden in der Richtlinie folgende Dimensionen der Unternehmensverantwortung unterschieden:

- $\quad$ Eigenverantwortung und innere organisatorische Ordnung;

- Verantwortung für den einzelnen Menschen und für die Menschlichkeit sowie Respekt für Menschenrechte;

- Verantwortung für das eigene Personal, für den Umgang mit Humanressourcen, für humane Arbeitsbedingungen, für Standards bezüglich des Arbeitsrechts und der Arbeitssicherheit sowie für gerechte Gewinnteilung;

- Verantwortung für die Umwelt, für den Umgang mit Naturressourcen, für nachhaltige Produktion und die Minimierung der Auswirkungen auf die Umwelt;

- Verantwortung für eine faire und ehrliche Abwicklung der Geschäfte, für gegenseitiges Vertrauen in Wirtschaftsbeziehungen und für fairen Handel;

- Verantwortung für den Kunden und den Schutz der Verbraucher;

- Verantwortung für die Lokalbevölkerung, für die Gemeinschaft und für ein gutes Leben der gesamten Menschheit, sowie für das Unternehmen als korporativen Bürger und guten Nachbarn.

Erklärungsversuch. Frankfurt am Main 2006; A. Schneider, R. Schmidpeter (Hrsg.), Corporate Social Responsibility - verantwortungsvolle Unternehmensführung in Theorie und Praxis. Berlin/Heidelberg 2012.

${ }^{11}$ Siehe hierzu den Beitrag von A. Kiepas in diesem Band; J. Jonker, W. Stark, S. Tewes, Corporate Social Responsibility und nachhaltige Entwicklung. Einführung, Strategie und Glossar.

Berlin/Heidelberg 2011; vgl. auch K. Michalski, Corporate Social Responsibility - ein Schritt zu einer Kultur der Nachhaltigkeit. In: Modern Management Review, Vol. XIX, No. 3(2014), S. 59-76. ${ }^{12}$ Vgl. A. Giddens, Klimatyczna katastrofa [Klimakatastrophe]. Warszawa 2010, S. 71f.(poln.).

${ }^{13}$ Vgl. Guidance on social responsibility, ISO 26000:2010. 
Die Grundlage der sozialen Verantwortung eines jeden Unternehmens auf all diesen Ebenen stellen die Wahrnehmung der Zusammenhänge eigener Wirtschaftsaktivitäten mit lebenswichtigen Interessen bestimmter Gruppen von Betroffenen, das Bewusstsein über die komplexe soziale „Einbettung“ des Unternehmenshandelns, seine sozialen Erfolgsbedingungen und die Fülle seiner sozialen Folgen, eine ehrliche Beurteilung eigener Praxen unter Berücksichtigung der Interessen aller Betroffenen und der Öffentlichkeit sowie die Bereitschaft, sich im Handeln auf die Güte- und Nutzenoptimierung für möglichst alle Beteiligten und Betroffenen zu orientieren, dar. Als notwendige Bedingungen der Möglichkeit, soziale Verantwortung im Wirtschaftshandeln richtig zu praktizieren, werden in der Richtlinie ISO26000 folgende Anforderungen genannt:

- Transparenz des Handelns, die u.a. zuverlässiges laufendes Informieren über aktuelle Vorgehen und Vorhaben, Motive, Wertvorstellungen und Präferenzen, gesetzte Ziele und eingesetzte Mittel und Ressourcen, den Umgang mit eigenen Human- und Materialressourcen sowie Umweltgütern, die Auswirkungen des eigenen Handelns auf die Umwelt sowie auf das Leben von Individuum und Gesellschaft, innere Entscheidungsprozesse, angenommene betriebseigene Verhaltensstandards und eingeführte Kontrollmechanismen für deren Befolgung, aber auch die im Falle ihrer Verletzung oder Missbrauchs drohenden Sanktionen und Konsequenzen, sowie die gewählte interne und externe Kommunikationskultur umfasst.

- Befolgung von ethischen Grundsätzen wie Berücksichtigung einer jeden Person und Respekt für ihre Menschenwürde sowie ihre lebenswichtigen Interessen, Beachtung der Menschenrechte, Gleichbehandlung unter vergleichbaren Bedingungen, Gerechtigkeit und Fairness, Einhalten von Versprechen und Erfüllen von Verpflichtungen etc., die in dem gesellschaftlichen Umfeld, in dem das konkrete Unternehmen agiert, anerkannt und praktiziert werden.

- Aufbau richtiger, beiderseitig zufriedenstellender und womöglich gewinnbringender Relationen mit allen Interessensubjekten und Betroffenen, Sensibilität für deren Bedürfnisse, Fürsorge und angemessenes Reagieren auf ihre Probleme, Gewährung des Rechts auf Mitsprache und Mitbestimmung durch Einbeziehung unterschiedlicher Interessenvertreter und der Betroffenen in Entscheidungsprozesse im Unternehmen.

- Pflegeabsoluter Legalität und höchsten Respekts gegenüber Gesetzen sowohl innerhalb des Unternehmens als auch in dessen Handlungsumfeld, Vermeiden von Handlungen, die bei eigenen Mitarbeitern und in der Öffentlichkeit den geringsten Verdacht auf irgendeinen Verstoß gegen das geltende Recht erwecken oder sie in dieser Hinsicht verunsichern könnten.

- Befolgung internationaler Verhaltensstandards und international geltender wirtschaftsethischer und kultureller Normen im Falle eines Mangels an entsprechenden nationalen Regelungen, etwa hinsichtlich Umwelt-, Sozial- und Sicherheitsstandards oder der Produktinformation.

- Weltweites, bedingungs- und kompromissloses Beachten von Menschenrechten, die in der Allgemeinen Erklärung der Menschenrechte (UNO, 1948) und in Folgedokumenten des internationalen Rechts fixiert sind. Wachen über das weltweite Respektieren von Menschenrechten und angemessenes Reagieren auf jeden Verstoß oder Missbrauch durch Verzicht auf Wirtschaftskooperationen mit den jeglicher 
Menschenrechtsverletzung Verdächtigen bzw. durch Rückzug aus den Märkten, in denen Menschenrechte nicht richtig geschützt werden, durch Boykott und WhistleblowingAktionen. ${ }^{14}$ In diesem Zusammenhang sind sozial sensible, verantwortliche und engagierte Unternehmen zu Tätigkeiten verpflichtet, die weit über den normalen alltäglichen Wirtschaftsbetrieb hinausgehen und auf die Absicherung fundamentaler politischer und bürgerlicher Rechte und Freiheiten wie auch der wichtigsten sozialen, ökonomischen und kulturellen Rechte, wie Recht auf Arbeit, selbstständigen Lebensunterhalt und soziale Absicherung im Alter und im Falle einer anderen Unfähigkeit zur Erwerbstätigkeit, Recht auf Gesundheitsschutz oder Zugang zur Bildung, Kultur und Möglichkeiten einer menschengerechten Sozialisierung abzielen. ${ }^{15}$

Über die vordergründigen Ziele von CSR-Initiativen hinaus kann auf zahlreiche Nebenfunktionen dieser Aktivitäten, die für die Gesellschaft keine geringere Bedeutung haben, hingewiesen werden. Diese bestehen im Wecken des öffentlichen Bewusstseins über die Dimensionen der Unternehmensverantwortung, die sich aus dem gesellschaftlichen Auftrag und der gesellschaftlichen Handlungslizenz ergeben, in der Verbreitung der Grundhaltung einer auf menschlicher Solidarität und Fürsorge fundierten Langzeit- und „Langstreckenverantwortung“, in der Orientierungsleistung, die den Einzelnen etwa als Konsumenten bei der täglichen „Qual der Wahl“ entlasten und die Gemeinschaft bei Entscheidungsprozessen zur Festsetzung der Rahmenbedingungen für Unternehmenshandeln unterstützen soll, in der gesellschaftlichen Legitimierung sowohl einzelner betriebseigener Wirtschaftsentscheidungen als auch der gesamten Geschäftsaktivität eines Unternehmens, in der Koordinierung der Zusammenarbeit mit anderen Akteuren des ersten (öffentliche Institutionen), zweiten (Wirtschaftsorganisationen) und dritten (zivilgesellschaftliche Organisationen) Sektors bei der Etablierung neuer Standards sozial verantwortlichen Wirtschaftens und einer allgemeinen Gemeinwohlorientierung, in Verhinderung von Unfällen, Katastrophen und Krisensituationen, die infolge von Nonchalance, Unbekümmertheit und Verantwortungslosigkeit zustande kommen, in Vorbeugung von sozialen Konflikten innerhalb und außerhalb des Unternehmens, im Schutz des Unternehmens vor eventuellen Ansprüchen Dritter und im Schutz der Mitarbeiter, die selber moralisch richtig handeln und angesichts erkannter Missstände (Missbräuche, Defraudationen und Verschwendungen, Korruptionsfälle, Verstöße gegen Gesetze, wirtschafts- und

\footnotetext{
${ }^{14}$ „Whistle-blowing“ besteht primär im demonstrativen Informieren der Öffentlichkeit über Missstände (Gesetzesverstoße, Straftaten, Unterschlagen von Gefahren, Bagatellisierung von Schadensfällen etc.) in Betrieben. Parallel dazu können und sollen auch von Verantwortlichen, die außerhalb eines Unternehmens agieren (Politik, konkurrierende Unternehmen oder Organisationen der Zivilgesellschaft)entsprechende Missstände öffentlich gemacht werden, die etwa für die Entscheidungen von potenziellen Kooperationspartnern oder Verbrauchern relevant sein können; siehe hierzu K. Leisinger, Whistleblowing und Corporate Reputation Management, München 2003; K. Michalski, Główne zagadnienia etyki inżynierskiej [Grundprobleme der Ingenieursethik]. In: M. Małek, E. Mazurek, K. Serafin (Hrsg.), Etyka i technika. Etyczne, społeczne i edukacyjne aspekty działalności inżynierskiej [Ethik und Technik. Ethische, soziale und bildungsmäßige Aspekte des Ingenieurshandelns]. Wrocław 2014, S. 58ff. (poln.).

${ }^{15}$ Vgl. J. Stec-Rusiecka, Wpływ społecznej odpowiedzialności na poprawę jakości życia społeczeństwa [Der Einfluss sozialer Verantwortung auf die Verbesserung der Lebensqualität der Gesellschaft], In: Modern Management Review, Vol. XVIII (2/2013), S. 86f. (poln.).
} 
berufsethische Standards oder betriebsinterne Statuten)in guter Absicht Alarm schlagen, ${ }^{16}$ und nicht zuletzt in der Minimierung oben erwähnter Missstände durch Einführung eines komplexen, ganzheitlichen unternehmensethischen Programms, das neben inhaltlichen Elementen wie Ethikkodizes und Verhaltensregeln auch konkrete Kontroll- und Sanktionsmechanismen in Form von Infolinien, internen und externen Audits, Ethikkommissionen und -beauftragten, Mitarbeiterrankings, Prämien für verantwortungsvolles Verhalten etc. vorsieht.

Auf der Ebene der Unternehmensführung und des Managementsystems (1) sind sowohl eine Einbeziehung der CSR-Grundsätze in das betriebsinnere ManagementRegelwerk, die auf einer maximalistischen Annahme basiert, dass ein effektives Engagement für die Verbesserung der gesellschaftlichen Lebensqualität und die Manifestierung sozialer Verantwortung zur erheblichen Gewinnoptimierung u.a. durch Motivations- und Leistungssteigerung seitens der Arbeitskräfte, durch Verbesserung der Atmosphäre der Wirtschaftskooperation und durch eine allgemeine Verstärkung des Vertrauens im Unternehmen und um Unternehmen herum beiträgt, als auch eine Verbesserung des Managements in den wichtigsten CSR-relevanten Bereichen der Unternehmensaktivität möglich. Wichtige Instrumente, auf die im Rahmen eines intelligenten und sozial verantwortlichen Managements gerne zurückgegriffen wird, sind Unternehmenskodizes, das Delegieren bestimmter, im Rahmen der bestehenden Management-Strukturen verfügbarer Humanressourcen zur Realisierung der CSRAufgaben, zu derer Überwachung und zur ständigen Verbesserung diesbezüglicher Maßnahmen, das Einführen eines entsprechenden Anreiz-Systems zur Förderung des CSR-Engagements und der Kreativität in der Suche nach neuen Lösungen und Anwendungsfeldern, das Aufbauen eines inneren Warnsystems zum Erkennen und Verhindern von Missständen in CSR-relevanten Handlungsbereichen sowie eine periodische Durchsicht aller Managementprozesse, deren Bewertung und ständige Verbesserung.

Auf der Ebene der Beschäftigungspolitik (2), die sowohl die weitgehend gesetzlich geregelten Anforderungen des Arbeitsschutzes, der Arbeitssicherheit und der Hygiene als auch unternehmenseigene Prinzipien und Verfahren der Rekrutierung, Beförderung, Bestrafung und Entlassung von Mitarbeitern, die Regelungen hinsichtlich der Arbeitslohnpolitik, der Schulung und Weiterbildung der Firmenkader, des Dialogs mit Gewerkschaften und anderen Mitarbeiterorganisationen etc. umfasst, sollten in erster Linie Interessen und Bedürfnisse der schwächeren, benachteiligten oder unterrepräsentierten Beschäftigtengruppen wie Frauen, bestimmte Altersgruppen oder spezielle sozial profilierte Gruppen wie Alleinerziehende, Träger und Alleinernährer von kinderreichen Haushalten oder Bewohner von Gebieten, die besonders gesundheits- oder umweltschädlichen Auswirkungen der Produktion oder anderer Unternehmensaktivitäten ausgesetzt sind, berücksichtigt werden. Im Sinne der sozialen Gerechtigkeit als Kompensation ${ }^{17}$ sollten im Rahmen der CSR-Strategie auf dieser Ebene bestehende Ungleichstellungen, Benachteiligungen und Schädigungen durch angemessene „Privilegien“ ausgeglichen werden. Das gilt primär für Missstände, die im

\footnotetext{
${ }^{16}$ Zur aktuellen Diskussion um einen rechtlichen Whistleblower-Schutz in Deutschland siehe D. Deiseroth, Zur gesetzlichen Neuregelung des Schutzes von Whistleblowern/Informanten. Bundestag Ausschussdrucksache 16 (10) 850 - I. Berlin 2008.

${ }^{17}$ Vgl. J. Rawls, Theory of Justice. Boston 1971.
} 
Zusammenhang mit dem Unternehmenshandeln in der Vergangenheit entstanden sind oder immer noch entstehen.

Auf der Ebene des Umweltmanagements (3) haben Unternehmen nahezu uneingeschränkte Möglichkeiten, sich für ein besseres Leben von Mensch und Gesellschaftheute und morgen einzusetzen. Sie können umfangreich umweltschädliche Folgen ihres Wirtschaftshandelns auf komplexe Weise erkennen und systematisch minimieren, durch einen sparsamen Umgang mit Ressourcen und durch Steigerung ihrer Nutzungseffizienz ein nachhaltiges Ressourcenmanagementsystem aufbauen, durch Reduzierung von direkten Emissionen und der Erhöhung der Energieintensität und effizienz ihrer Produktions-, Verladungs- und Vertriebsprozesse das Klima schonen, durch Verbesserung ihrer Logistik, Rationalisierung der Lieferketten, Vermeidung unnötiger Mobilisierung von Waren und Personen und durch Optimierung der Nutzung von Produktions-, Lager-, Büro- und Handelsflächen zur erheblichen Umweltentlastung beitragen und durch Verbesserung ihrer Informationspolitik und der Kommunikation mit Kunden und Verbrauchern im Hinblick auf die Behandlung von Reklamationen, Produktkennzeichnung und Produktinformation und eine ausführliche, alle umweltschutzrelevanten Produktnutzungsaspekte umfassende Gebrauchsanweisung, die komplexe Informationen über alle Auswirkungen der eigenen Produkte auf die Umwelt in allen Phasen ihres Lebenszyklus, über die umweltfreundlichste Nutzung und die aus der Sicht des Umweltschutzes optimale Entsorgung der verbrauchten oder ungebrauchten Produkte enthalten, zum nachhaltigen Konsum beitragen. Im Rahmen ihrer Initiativen für die Umweltschonung sollten Unternehmen nicht nur Verantwortung für Entscheidungen und das Verhalten ihrer Kunden übernehmen, sondern auch ihre Kontrahenten und Kooperationspartner (Dienstleister, Zulieferer, Handelsvertreter etc.) zur Beachtung und Befolgung der gleichen Standards der Umweltnutzung mobilisieren. Ein intelligentes und sozial verantwortliches Umweltmanagement betrachtet die Form der Nutzung von Umweltgütern und das $\mathrm{Ma} ß$ des ökologischen Bewusstseins und Engagements eines Unternehmens als Prüfstein für dessen soziale, gesellschaftliche Glaubwürdigkeit. Die Unternehmen sollten aufpassen, dass dieser Prüfstein nicht zu ihrem Stolperstein wird.

Auf der Ebene der Interaktionen mit Akteuren aus dem öffentlichen Umfeld (staatliche Institutionen und zivilgesellschaftliche Organisationen) und der Zusammenarbeit mit dem unmittelbaren und weiteren Wirtschaftsumfeld (4) verpflichtet die Philosophie sozialer Verantwortung zu einer Beschränkung ausschließlich auf legale, ehrliche, ethisch integrale, konsequente und transparente Geschäftspraxen, die auf den vom Umfeld anerkannten und geforderten Standards der Wirtschaftsethik basieren und auf eine niveauhohe Managementkultur, das Verfügen über eine reife und durchdachte Handlungsstrategie, einen hohen Grad innerer sozialer und organisatorischer Integrität und eine hohe gesellschaftliche Interaktivität und Sensibilität für die Reputationsaspekte hin deuten. Ein vorsichtiges und verantwortungsvolles politisches Engagement des Unternehmens, eine konsequente, kompromisslose und engagierte Haltung gegenüber Korruption, die ständige Bereitschaft, Zeugnisse überseine Ehrlichkeit und Fairness, seine Ziele, Werte und Grundsätze in allen seinen Wirtschaftsbeziehungen zu geben, der Wille, zum Vorbild für andere zu werden und dadurch bei den Wirtschafts- und Kooperationspartnern, mit denen das Unternehmen größere Einheiten, Lieferketten und Wirtschaftsallianzen bildet, ähnliche Ambitionen zu erwecken - alle diese Aktivitäten beweisen, dass ein Unternehmen seine gesellschaftliche Dienstbarkeit seriös betrachtet und dass es ernsthaftes Interesse nicht nur daran hat, wie es von der Umgebung 
wahrgenommen wird, und dass seine CSR-Initiativen weit mehr bedeuten als lediglich gesellschaftliches Alibi-Beschaffung und Blue- oder Greenwashing.

$\mathrm{Zu}$ den wichtigsten Formen und Manifestationen des sozialen Engagements eines Unternehmens auf der Ebene der Relationen mit Kunden und Verbrauchern (5) gehören neben den erwähnten Initiativen, die im Bereich der Umweltverantwortung auf einen nachhaltigen Konsum abzielen, u.a. die Sorge für die Sicherheit und Zufriedenheit der Kunden durch die Sicherung angemessener Qualität, Funktionsfähigkeit, Zuverlässigkeit und Wirtschaftlichkeit eigener Produkte und durch ausführliche Information über die im Hinblick auf Nachhaltigkeit, Beständigkeit, Sicherheit und Effektivität optimale Nutzung des Produktes. Die Gewährleistung der Verbrauchersicherheit geschieht in erster Linie durch einen bewussten Verzicht auf Lösungen, die real (Eintrittswahrscheinlichkeit eines Schadensereignisses bekannt) oder hypothetisch (Eintrittswahrscheinlichkeit eines Schadensereignisses unbekannt) das Leben oder die Gesundheit des Verbrauchers oder Dritter gefährden. Sind die entsprechenden Risiken, die sich aus dem Besitz oder Gebrauch der Unternehmensprodukte ergeben, nicht zu vermeiden, muss der Verbraucher über diese ausführlich informiert werden. Neben Sorge für die Sicherheit des Verbrauchers sind auch eine faire Preisgestaltung, der Verzicht auf ethisch fragwürdige Formen der Persuasion (Zurede), Manipulation, Täuschung, Erpressung und Erzwingung etwa durch aggressive Werbung, gezielte Inkompatibilität eigener Erzeugnisse mit Produkten anderer Hersteller (wie es über lange Zeit bei den Handy-Ladegeräten der Fall war) oder entsprechendes Arrangement der Verkaufssituation, kundenfreundliche Abwicklung von Reklamationen und gewissenhafte, zuverlässige Erfüllung aller Garantieleistungen und nicht zuletzt eine angemessene Entschädigung und Kompensation im Falle eines durch das Unternehmen verschuldeten Kundenschadens Kernelemente der Verantwortung gegenüber dem Verbraucher. $\mathrm{Zu}$ anderen wichtigen Anforderungen, die sich für das Unternehmen aus sozialer Verantwortung in den Relationen mit Kunden ergeben, gehören auch vollständiger und bedingungsloser Respekt für ihre Menschenwürde und die Achtung der Kundenprivatheit. Diese verbieten u.a. jegliche Invigilation (Bespitzelung), Nutzung ihrer geschützten Personaldaten ohne ihre Kenntnis und Zustimmung, Verletzung ihrer sonstigen Privatrechte sowie Verwendung von Werbungsinhalten, die von einzelnen Verbrauchern oder von Verbrauchergruppen als beleidigend, diskriminierend oder demoralisierend empfunden werden könnten.

Schließlich erfordert die Philosophie des sozialen Engagements und der gesellschaftlichen Verantwortung von Unternehmen auf der Ebene der Relationen mit der Öffentlichkeit (6) ein ständiges Manifestieren der gesellschaftlichen Zugehörigkeit des Unternehmens - sozial verantwortliche Unternehmen zeigen der Gesellschaft stets, dass sie sich als Teil dieser Gesellschaft sowie als produktives und multidimensionalen Nutzen bringendes Glied verstehen. Die Identifizierung von Rollen, die sich aus solcher gesellschaftlicher Zugehörigkeit ergeben, hängt vom Verständnis dessen ab, was aus einer Menge von Menschen eine Gesellschaft macht, was die Quelle ihrer Sicherheit und ihres Zusammenhalts ist und was ihr Zusammenleben gut und noch besser macht. Diese soziale Einbettung und Verwurzelung der Wirtschaftsunternehmen stellt ein interessantes, schnell wandelndes Forschungs- und Versuchsfeld dar, und zwar aus dem Grund, dass wir heute mit einer enormen strukturellen Komplexität und unterschiedlichen territorialen Skalen des Unternehmenswirkens konfrontiert sind. Angesichts dieser Komplexität und Diversität ist es sehr schwierig, universelle Rezepte für gelungene Relationen mit der Öffentlichkeit und für konstruktive, für beide Seiten nützliche und zufriedenstellende 
Interaktionen der Unternehmen mit lokalen Gemeinschaften zu formulieren. Sicherlich sind diese ohne einen aktiven und für die Bevölkerung sichtbaren Beitrag der Unternehmen zum „,besseren Leben“ einer Gesellschaft, insbesondere auf den Feldern, die vom Staat allmählich verlassen werden, wie Kultur, Bildung und Wissenschaft, Massensport und öffentliche Gesundheit, Umweltschutz oder soziale Absicherung. In der Gesellschaft gibt es so viele unbefriedigende Bedürfnisse, dass es den Unternehmen an Ideen für ein korporatives Volontariat und für diesbezügliche CSR-Initiativen sicherlich nicht mangeln wird. Es ist die Entscheidung jedes Unternehmens, ob es das Leben einer Gesellschaft rein zufällig, absichtslos, unreflektiert und unkontrolliert beeinflussen will oder lieber planmäßig, intentional, bewusst und gesteuert im Rahmen einer eigenen, gut durchdachten CSR-Strategie zum besseren Leben für alle beiträgt. Nur im letzteren Fall werden die Unternehmensaktivitäten nachhaltig die geforderten Vorteile für beide Seiten bringen und für das gegenseitige, für eine fruchtbare langfristige Kooperation erforderliche Vertrauen sorgen. ${ }^{18}$

Die oben strukturierten Dimensionen und Aspekte der sozialen Verantwortung werden von Unternehmen unterschiedlich erkannt und für die gesellschaftliche Reputationsbildung genutzt. Doch immer mehr Unternehmen entdecken im gesellschaftlichen Engagement eine Chance, wirtschaftlichen Zielen einen höheren, mit den sonstigen Bereichen menschlichen Lebens korrespondierenden Sinn zu geben. Laut einer Befragung aus dem Jahre 2008 sahen fast $70 \%$ der Unternehmen in CSR-Initiativen einen wichtigen Renditefaktor. ${ }^{19}$ Vor diesem Hintergrund bekommt die von Wirtschaftsethikern über Jahrzehnte entwickelte Vision eines umweltfreundlichen, menschen- wie gesellschaftsdienlichen und trotzdem noch rentablen Wirtschaftshandelns ${ }^{20}$ dank einer richtigen Operationalisierung des CSR-Leitbildes eine etwas konkretere Gestalt. Dabei wurde am Beispiel der Handlungs- und Gestaltungspotenziale von Großunternehmen gezeigt, wie sich die beiden Träger der modernen Kultur - Nachhaltigkeit und soziale Verantwortung - gegenseitig bedingen. So wird das Erreichen der Ziele nachhaltiger Entwicklung ohne gesellschaftliche Verantwortung von Unternehmen und ohne ihr soziales wie ökologisches Engagement nur schwer möglich sein.

\footnotetext{
${ }^{18}$ Vgl. J. Stec-Rusiecka, op. cit., S. 86-88.

${ }^{19}$ G. Pohle, J. Hittner, Attaining Sustainable Growth through Corporate Social Responsibility. New York 2008, S. 3.

${ }^{20}$ Ausführlicher dazu in A. Löhr, Unternehmensethik und Betriebswirtschaftslehre. Untersuchungen zur theoretischen Stützung der Unternehmenspraxis. Stuttgart 1991; H. Steinmann (Hrsg.),

Unternehmensethik. 2. Aufl. Stuttgart 1991; K. Homann, F. Blome-Drees, Wirtschafts- und Unternehmensethik. Göttingen 1992; K. Homann, Moral in den Funktionszusammenhängen der modernen Wirtschaft. Zwei Beiträge zur Wirtschaftsethik unter Wettbewerbsbedingungen. Stuttgart 1993; H. Steinmann, A. Löhr, Grundlagen der Unternehmensethik. 2. Auf. Stuttgart 1994; H. G. Nutzinger (Hrsg.), Ökonomie der Werte oder Werte in der Ökonomie. Unternehmenskultur in genossenschaftlichen, alternativen und traditionellen Betrieben. Marburg 1996; H. G. Nutzinger (Hrsg.), Wirtschafts- und Unternehmensethik. Kritik einer neuen Generation. Zwischen Grundlagenreflexion und ökonomischer Indienstnahme. München 1999; P. Ulrich, Integrative Wirtschaftsethik. Grundlagen einer lebensdienlichen Ökonomie. 3. Aufl. Wien 2001.
} 


\section{LITERATUR}

[1] Abriszewski K., Interpretacja i etyka dalekiego zasieggu [Interpretation und Ethik einer Langzeit- und Langstreckenverantwortung]. In: A. F. Kola, A. Szahaj (Hrsg.), Filozofia i etyka interpretacji [Philosophie und Ethik der Interpretation]. Kraków 2007, S. 277-288 (poln.)

[2] Banse G., Kiepas A. (Hrsg.), Nachhaltige Entwicklung: Von der wissenschaftlichen Forschung zur politischen Umsetzung. Berlin 2005

[3] Banse G., Janikowski R., Kiepas A. (Hrsg.), Nachhaltige Entwicklung transnational. Sichten und Erfahrungen aus Mitteleuropa. Berlin 2011

[4] Banse G., Nelson G. L., Parodi O. (eds.), Sustainable Development - the Cultural Perspective. Concepts - Aspects - Examples. Berlin 2011

[5] Barker D., Mander J., ABC globalizacji [ABC der Globalisierung]. Międzynarodowe Forum ds. Globalizacji [Internationales Forum für Globalisierung]. Łódź 2003, S. 23 (poln.)

[6] Bhagwati J., In Defense of Globalization. New York 2004

[7] Bińczyk E., Technonauka w społeczeństwie ryzyka. Filozofia wobec niepożadanych nastepstw praktycznego sukcesu nauki [Technowissenschaft in der Risikogesellschaft. Die Philosophie angesichts der unerwünschten Folgen des praktischen Erfolgs der Wissenschaft]. Toruń 2013 (poln.)

[8] Bowen H.R., Social responsibility of the businessman. New York 1953.

[9] Deiseroth D., Zur gesetzlichen Neuregelung des Schutzes von Whistleblowern/Informanten. Bundestag Ausschussdrucksache 16 (10) 850 I. Berlin 2008

[10]Die EU-Richtlinie 2014/95/EU vom 22. Oktober 2014 zur CSRBerichterstattung

[11] Giddens A., Klimatyczna katastrofa [Klimakatastrophe]. Warszawa 2010 (poln.)

[12] Grünbuch Europäische Rahmenbedingungen für die soziale Verantwortung der Unternehmen. $\mathrm{KOM}(2001) 366$ endgültig

[13]Guidance on social responsibility, ISO 26000:2010

[14] Hiß S., Warum übernehmen Unternehmen gesellschaftliche Verantwortung? Ein soziologischer Erklärungsversuch. Frankfurt am Main 2006

[15]Homann K., Moral in den Funktionszusammenhängen der modernen Wirtschaft. Zwei Beiträge zur Wirtschaftsethik unter Wettbewerbsbedingungen. Stuttgart 1993

[16] Homann K., Blome-Drees F., Wirtschafts- und Unternehmensethik. Göttingen 1992

[17] Jonker J., Stark W., Tewes S., Corporate Social Responsibility und nachhaltige Entwicklung. Einführung, Strategie und Glossar. Berlin/Heidelberg 2011

[18] Leisinger K., Whistleblowing und Corporate Reputation Management, München 2003

[19]Löhr A., Unternehmensethik und Betriebswirtschaftslehre. Untersuchungen zur theoretischen Stützung der Unternehmenspraxis. Stuttgart 1991

[20] Michalski K., Corporate Social Responsibility - ein Schritt zu einer Nachhaltigkeitskultur. In: Modern Management Review, Vol. XIX, No. 3(2014), S. 59-76 
[21] Michalski K., Główne zagadnienia etyki inżynierskiej [Grundprobleme der Ingenieursethik]. In: M. Małek, E. Mazurek, K. Serafin (Hrsg.), Etyka $i$ technika. Etyczne, społeczne i edukacyjne aspekty działalności inżynierskiej [Ethik und Technik. Ethische, soziale und bildungsmäßige Aspekte des Ingenieurshandelns]. Wrocław 2014, S. 31-60 (poln.)

[22] Nutzinger H. G. (Hrsg.), Ökonomie der Werte oder Werte in der Ökonomie. Unternehmenskultur in genossenschaftlichen, alternativen und traditionellen Betrieben. Marburg 1996

[23] Nutzinger H. G. (Hrsg.), Wirtschafts- und Unternehmensethik. Kritik einer neuen Generation. Zwischen Grundlagenreflexion und ökonomischer Indienstnahme. München 1999

[24] Parodi O., Banse G., Schaffer A. (Hrsg.), Wechselspiele: Kultur und Nachhaltigkeit. Annäherungen an ein Spannungsfeld. Berlin 2010

[25] Pohle G., Hittner J., Attaining Sustainable Growth through Corporate Social Responsibility. New York 2008

[26] Rawls J., Theory of Justice. Boston 1971

[27] Schneider A., Schmidpeter R. (Hrsg.), Corporate Social Responsibility verantwortungsvolle Unternehmensführung in Theorie und Praxis. Berlin/Heidelberg 2012

[28] Steinmann H. (Hrsg.), Unternehmensethik. 2. Aufl. Stuttgart 1991

[29] Steinmann H., Löhr A., Grundlagen der Unternehmensethik. 2. Auf. Stuttgart 1994

[30] Stec-Rusiecka J., Wpływ społecznej odpowiedzialności na poprawę jakości życia spoleczeństwa [Einfluss der sozialen Verantwortung auf die Verbesserung der Lebensqualität der Gesellschaft]. In: Modern Management Review, Vol. XVIII No. 2(2013), S. 85-91 (poln.)

[31] Ślęzak-Gładzik I., Corporate Social Responsibility (CSR) jako koncepcja porzadkujaca relacje między biznesem a społeczeństwem [CSR als ein die Beziehungen zwischenWirtschaft und Gesellschaft ordnendes Konzept]. In: Modern Management Review, Vol. XVIII, No. 2(2013), S. 113-125 (poln.)

[32] Ulrich P., Integrative Wirtschaftsethik. Grundlagen einer lebensdienlichen Ökonomie. 3. Aufl. Wien 2001.

\section{RENTOWNA, PRZYJAZNA DLA ŚRODOWISKA I SPOLECZNIE UŻYTECZNA DZIAŁALNOŚĆ GOSPODARCZA JEST MOŻLIWA. WIĘCEJ ZRÓWNOWAŻONOŚCI DZIEKI LEPSZEJ OPERACJONALIZACJI SPOLECZNEJ ODPOWIEDZIALNOŚCI PRZEDSIĘBIORSTW}

Co prawda pojęcie „społecznej odpowiedzialności przedsiębiorstw“ robi dzisiaj furorę w publicznej dyskusji, jest jednak używane bardzo inflacyjnie, co na dłuższą metę nie służy właściwym celom CSR. Równocześnie w kontekście wszechobecnych sloganów wzywających do zrównoważonego rozwoju postuluje się głębokie przewartościowania w biznesie - „remoralizację” i „resocjalizację”. Wokół pytania, na czym polega społecznie odpowiedzialna przedsiębiorczość, trwają gwałtowne spory. Konsekwencją tej semantycznej dezorientacji są coraz głośniej zgłaszane postulaty rezygnacji z pojęcia CSR jako nieprzydatnego lub wprowadzającego w błąd i zastąpienie go bardziej zróżnicowanymi określeniami. W przedłożonym artykule podjęto próbę obrony pojęcia CSR i pokazania, że nie tylko $\mathrm{z}$ punktu widzenia etyki społecznej, ale także $\mathrm{z}$ punktu widzenia nauk 
ekonomicznych za utrzymaniem tego pojęcia przemawiają dobre racje. Główne tezy argumentacji to: zrównoważona, przyjazna dla środowiska i społecznie użyteczna działalność gospodarcza jest możliwa. Na płaszczyźnie przedsiębiorstwa droga do niej wiedzie poprzez całą pełnię możliwych inicjatyw z obszaru CSR, których jednak przedsiębiorstwa jeszcze w pełni nie dostrzegają. Na podstawie wytycznej ISO 26000 idea społecznej odpowiedzialności przedsiębiorstw została zoperacjonalizowana na sześciu poziomach: (1) zarządzania organizacją, (2) polityki zatrudnienia, (3) zarządzania środowiskowego, (4) interakcji $\mathrm{z}$ otoczeniem biznesowym, (5) relacji $\mathrm{z}$ klientami i konsumentami oraz (6) interakcji $\mathrm{z}$ otoczeniem publicznym i ze społecznościami sąsiedzkimi. Dzięki właściwej operacjonalizacji CSR wizja zrównoważonej działalności gospodarczej przybiera nieco konkretniejsze kształty.

Słowa kluczowe: zrównoważony rozwój, społeczna odpowiedzialność przedsiębiorstw, operacjonalizacja, etyka przedsiębiorstw.

PROFITABLE, ENVIRONMENTALLY FRIENDLY AND SOCIALLYSERVING MANAGEMENT IS POSSIBLE. MORE SUSTAINABILITY THROUGH BETTER OPERATIONALIZATION OF CORPORATE SOCIAL RESPONSIBILITY

While making the term "corporate social responsibility" in the public today a sensation, unfortunately it is still used very inflationary, what gives a few benefits to the CSR concern in the long run. At the same time in context of sustainability slogans there are profound transformations of values: re-moralization and re-socialization of business postulated. What is the corporate social responsibility precisely, is highly controversial. As a result of this semantic chaos, the resignation of the use of the CSR term as useless and misleading is postulated. This term begins to be replaced with more specific terms. In this paper an attempt is made to defend this concept and show, that there are not only socio-ethical, but also economic good reasons to keep it upright. The central thesis of the reflections are: sustainable, environmentally friendly and socially-serving management is possible. At the level of the company, the path leads to a whole range of possible CSR activities, that are not yet fully recognized by the company. On the basis of given in the standard ISO 26000 orientation, the corporate social responsibility can be operationalized on six levels: (1) the management and organization, (2) employment policy, (3) environmental and resource management, (4) the interactions with the economic environment, (5) the relations with customers, and (6) the interactions with the public and local communities. Thanks to the CSR operationalization, the vision of the sustainable business activities takes on a more concrete form.

Keywords: sustainability, corporate social responsibility, operationalization, business ethics.

DOI:10.7862/rz.2014.hss.42

Przesłano do redakcji: czerwiec 2014

Przyjęto do druku: październik 2014 